\title{
Autofluorescent Imaging in Patients With Peritoneal Carcinomatosis
}

Surgical Innovation

2014, Vol. 21 (2) 187-193

(C) The Author(s) 2013

Reprints and permissions:

sagepub.com/journalsPermissions.nav DOI: $10.1|77 / 15533506 / 34951| 4$

sri.sagepub.com

\author{
Philipp von Breitenbuch, MD', Tonia Jeiter', Stephan Schreml, MD',

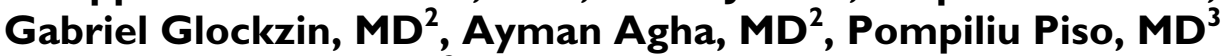 \\ and Hans J. Schlitt MD ${ }^{2}$
}

\begin{abstract}
Background and objectives. Autofluorescence imaging (AFI) is mainly used to detect (pre)cancerous colorectal and pulmonal lesions. This is the first report establishing the feasibility of AFI in patients with peritoneal carcinomatosis (PC). Methods. This is a prospective analysis of 10 patients undergoing conventional white-light laparoscopy (WL) and AFI for PC of different gastrointestinal tumors and I ovarian cancer. Before taking biopsies, suspicious peritoneal lesions were first detected by $\mathrm{WL}$ and then investigated by AFI. The intraoperative findings were photographed and then correlated with histological results. Results. Conventional WL and AFI evaluation was successful in all patients. A total of 38 biopsies were taken. The neoplasm detection rate under $\mathrm{WL}$ was $66 \%$ and increased to $86 \%$ when using AFI. The positive tumor detection rate was slightly higher in low AF lesions ( 83 vs $88 \%$ ) and higher in tumor nodules $(94 \%)$ than in flat peritoneal lesions $(75 \%)$. For tumor nodules, the sensitivity was $94 \%$, and the specificity was $100 \%$. For flat lesions, the sensitivity was $75 \%$ and specificity $50 \%$. Conclusions. We demonstrate the feasibility and effectiveness of AFI in patients with PC.
\end{abstract}

\section{Keywords}

visualization, laparoscopy, tumor

\section{Introduction}

Changes of the peritoneum can range in biological behavior from benign to highly malignant. Peritoneal carcinomatosis (PC) is the most common secondary tumor affecting the peritoneal cavity. ${ }^{1}$ Arising from malignant tumors of the gastrointestinal tract or the ovary, PC is associated with a poor prognosis. Cytoreductive surgery (CRS) followed by hyperthermic intraperitoneal chemotherapy (HIPEC) seems to be a treatment option for a group of highly selected patients suffering from PC, resulting in a significantly improved median survival., ${ }^{2,3}$ Penetration of chemotherapeutic molecules during HIPEC is limited to 1 to $2 \mathrm{~mm}$ in depth. ${ }^{4}$ Therefore, resection of all tumor deposits greater than $2 \mathrm{~mm}$ in diameter is essential. Optimal patient selection is mandatory, including early recognition and accurate assessment of the extent of PC.

For the diagnosis of PC, laparoscopy and biopsies of abnormal-appearing peritoneum are often part of the diagnostic workup. Laparoscopic detection of early malignant lesions on the peritoneal surface can be difficult because these foci can be subtle and flat. Because of limitations of conventional white-light laparoscopy (WL), it is frequently impossible to distinguish between malignant and benign peritoneal lesions. Improved imaging techniques may help in more accurately predicting the histology of peritoneal lesions. The combination of autofluorescence imaging (AFI) and laparoscopy might be a promising step in this direction.

AFI is based on the fact that nonneoplastic and neoplastic tissues have different characteristics regarding their short-wavelength autofluorescence. ${ }^{5,6}$ This is based on differences in the proportion of endogenous fluorophores (eg, tryptophan, collagen, and mitochondria) between normal and malignant tissue, resulting in a change in autofluorescent emission spectra. ${ }^{7-11}$ The epithelial thickness of dysplastic/malignant tissue also influences autofluorescence emission.

AFI has been implemented in several medical fields. It is currently used for the detection of dysplastic lesions in

\footnotetext{
'Elblandklinikum Riesa, Riesa, Germany

${ }^{2}$ University Medical Center Regensburg, Regensburg, Germany

${ }^{3}$ Krankenhaus der barmherzigen Brüder, Regensburg, Germany

Corresponding Author:

Philipp von Breitenbuch, Elblandklinikum Riesa, Klinik für Allgemein-, Viszeral- und Gefäßchirurgie, Weinbergstrasse 8, 01589 Riesa,

Germany.

Email: philipp.breitenbuch@elblandkliniken.de
} 
Table I. Characteristics of the Patients.

\begin{tabular}{|c|c|c|c|c|c|}
\hline Patient & Sex & Tumor Entity & Surgery Prior to Our DL & TNM Grading & Last Chemotherapy Prior to DL \\
\hline 1 & $\mathrm{~F}$ & GC & $\mathrm{DL}+$ biopsy & G3 & FLOT \\
\hline 2 & $\mathrm{~F}$ & GC & $\begin{array}{l}\text { Resection of a stenotic } \\
\text { small-bowel loop }\end{array}$ & G3 & $\mathrm{DCF}$ \\
\hline 3 & $\mathrm{~F}$ & $\mathrm{CC}$ & Left hemicolectomy & pT3, pNI, M0, G3 & FOLFOX \\
\hline 4 & M & $\mathrm{CC}$ & Left hemicolectomy & $\mathrm{pT} 3, \mathrm{pN} 2, \mathrm{M} 0, \mathrm{G} 2$ & FOLFIRI + Avastin \\
\hline 5 & $\mathrm{~F}$ & $\begin{array}{l}\text { Mucinous cystadenoma, } \\
\text { appendix }^{\mathrm{a}}\end{array}$ & CCR-0/HIPEC & - & - \\
\hline 6 & $\mathrm{~F}$ & $\mathrm{CC}$ & - & G3 & FOLFOX \\
\hline 7 & $\mathrm{~F}$ & OC & - & $\mathrm{G} 3$ & - \\
\hline 8 & $M$ & GC & Gastrectomy & $\mathrm{pT} 2 \mathrm{~b}, \mathrm{pNI}, \mathrm{M0}, \mathrm{G} 3$ & FLOT \\
\hline 9 & $\mathrm{~F}$ & $\mathrm{CC}$ & Left hemicolectomy & $\mathrm{PT} 3, \mathrm{pNI}, \mathrm{M0}, \mathrm{G} 3$ & FOLFOX \\
\hline 10 & $M$ & $\mathrm{CC}$ & $\mathrm{DL}+$ biopsy & G3 & FOLFOX \\
\hline
\end{tabular}

Abbreviations: F, female; M, male; GC, gastric cancer; CC, colon cancer; OC, ovarian cancer; DL, diagnostic laparoscopy; CCR-0, complete macroscopic cytoreduction; HIPEC, hyperthermic intraperitoneal chemotherapy; FLOT, 5-fluorouracil, leukovorin, oxaliplatin, and docetaxel; DCF, docetaxel, cisplatin, 5-fluorouracil; FOLFOX, folinacid, 5-fluorouracil, oxaliplatin; FOLFIRI, folinacid, 5-fluorouracil, irinotecan; PC, peritoneal carcinomatosis.

${ }^{2}$ Because of a mucinous cystadenoma of the appendix, patient no. 5 had been initially treated with a CCR-0/HIPEC. The histological result from biopsies taken from the recurrent tumor confirmed PC of a peritoneal mucinous carcinomatosis of the intermediate type (PMCA-I). ${ }^{18}$

patients with inflammatory bowel disease ${ }^{12-15}$ and has been used for the detection of nonpigmented endometriotic lesions. ${ }^{7}$ The visualization of reduced autofluorescence has proven advantageous in the diagnosis of precancerous and cancerous lesions in the bronchial tract and the bladder. ${ }^{16,17}$

Given these findings, we used AFI during laparoscopy in patients with PC. The aim was to evaluate the feasibility of AFI-augmented laparoscopy in the diagnosis of PC from different tumor entities. We also wanted to study whether the use of AFI laparoscopy helps differentiate normal from pathological peritoneal tissue and whether it helps in directing the biopsies taken during exploration.

\section{Methods}

\section{Patients and Indications for Diagnostic Laparoscopy}

The study was conducted between September 2010 and February 2011 . The study population consisted of 3 men and 7 women. The median age was 53 years (range = 46-71 years). The median body mass index (BMI) was 24 (range 20-38).

Diagnostic laparoscopy was performed to confirm the clinically and/or radiologically suspected PC in 5 patients and to evaluate the distribution and potential resectability in all patients. One patient had cytological evidence of malignant ascites and 4 other patients had undergone a laparotomy or laparoscopy elsewhere for diagnostic purposes. Therefore, the diagnosis of PC was already established in these patients, but there was no complete description of the extent of the disease. Also, 8 patients had received systemic chemotherapy prior to presentation at our hospital. The primary origins of $\mathrm{PC}$ were gastric cancer $(n=3)$, colon cancer $(n=5)$, ovarian cancer $(\mathrm{n}=1)$, and suspected peritoneal recurrence of a benign mucinous cystadenoma of the appendix $(n=1)$. This patient had been treated 15 months before at our institution with a complete macroscopic cytoreduction (CCR-0) followed by an intraoperative HIPEC. The new histology from biopsies taken at diagnostic laparoscopy during this admission confirmed PC of a peritoneal mucinous carcinomatosis of the intermediate type (PMCA-I).$^{18}$ Patient characteristics are given in Table 1.

\section{Surgical Technique and AFI}

Laparoscopy and CRS/HIPEC are performed at our clinic by the same team. Laparoscopic evaluation was done always as a separate procedure before CRS. Because of the laparoscopic results, the upcoming surgery can better be discussed with the patient and the intraoperative and postoperative stress for the patient as well as the work load for the surgical team can be forecast more precisely.

All laparoscopies were performed by 1 experienced surgeon. A 10-mm trocar was placed after minilaparotomy at the umbilicus and a pneumoperitoneum of $15 \mathrm{~mm} \mathrm{Hg}$ was established. The laparoscope was inserted and a $5-\mathrm{mm}$ trocar was inserted under direct vision. Atraumatic grasping forceps were used for examination. In view of planned subsequent CRS and HIPEC, only minor adhesiolysis was performed. The laparoscope used was equipped for a 
Table 2. Matrix for the Calculation of Sensitivity and Specificity for All Biopsies Taken Under AFI Guidance.

\begin{tabular}{lll}
\hline & Patient With Tumor $(r p+f n), n=29$ & Patient Without Tumor $(f p+r n), n=8$ \\
\hline AFI tumor positive $(r p+f p), n=29$ & Right positive $(r p), n=25$ & False positive $(f p), n=3$ \\
AFI tumor negative $(f n+r n), n=9$ & False negative $(f n), n=4$ & Right negative $(r n), n=5$ \\
\hline
\end{tabular}

Abbreviation: AFI, autofluorescence imaging.

trimodal light source (Karl Storz GMBH\&CO.KG 78532, Tuttlingen, Germany). It was connected to a video processor (Karl Storz GMBH\&CO.KG 78532). One device of the laparoscope was used for conventional WL laparoscopy, the other for AFI. The third device can be used for fluorescence diagnostics after systemic administration of a photosensitizer, but this was not done during this study. In AFI mode, excitation was performed by blue light, and a high-pass filter (500-630 nm) was used to detect AF emission. In this setting, green $(528 \mathrm{~nm})$ and red light $(625 \mathrm{~nm})$ can pass through the filter, whereas blue light $(458 \mathrm{~nm})$ is blocked. When illuminated with blue light, dysplastic and malignant lesions appear purple. The sequentially detected AF images were integrated by a processor resulting in a real-time image. ${ }^{19,20}$

Flat and nodular areas were observed first by conventional WL, then via AFI. Before taking the biopsy, a photo of the region of interest was taken under WL and AFI to enable correlation of the intraoperative findings with the histological results.

Specimens were fixed in formalin, embedded in paraffin, cut, stained with hematoxylin and eosin, and examined by an experienced pathologist.

\section{Classification of Lesions Using AF}

AF intensity of the peritoneal lesions was optically determined by the color of the areas. As reported by van den Broek et al, ${ }^{21}$ tissue appearing green under AFI is usually healthy tissue. Purple lesions were considered as potentially malignant. Because of different color intensities of the purple lesions, we divided them into 2 groups. Light purple areas were regarded as having a high AF, suggesting that this tissue might have less pathological changes. By contrast, dark purple areas were regarded as potentially exhibiting major pathological changes. They were regarded as low AF. The intensity of the purple color under AFI was subjectively scored by the surgeon.

\section{Sensitivity and Specificity}

The matrix shown in Table 2 was used to calculate the sensitivity, specificity, positive predictive value, and negative predictive value. Histological results were compared with the results from WL and AFI laparoscopy.

\section{Ethical Considerations}

This prospective study was approved by the local ethics committee (No. 11-101-0282). Procedures were done in accordance with the Declaration of Helsinki.

\section{Results}

\section{Mortality and Morbidity}

Conventional WL and AFI evaluation was successful in all 10 patients. The mean operating time was 48 minutes (range $=25-72$ minutes). Laparoscopic abdominal exploration and tissue sampling were well tolerated. No significant blood loss was observed. One postoperative complication occurred in a patient with a BMI of 38 . This patient noticed some pain next to a trocar wound on postoperative day 2. Fascial closure of the abdominal wall proved to be insufficient, and the patient was reoperated on postoperative day 3 , and the defect was repaired. The patient was discharged on postoperative day 6 without further complications. All other patients were discharged on postoperative day 2 or 3 . The median length of hospital stay was 3 days.

\section{Histology and Tumor Entities}

A total of 38 laparoscopic biopsies were taken - at least 2 biopsies per patient (range $=2-6$ ). Histological validation was successful in 9 patients with clinical suspicion of PC. In 1 patient with a suspected recurrence of a benign mucinous cystadenoma of the appendix, the biopsies surprisingly showed a PMCA-I. ${ }^{18}$ Apart from this patient, the tumor-positive biopsies always corresponded with the histology of the primary tumor.

Table 3 shows the morphology of lesions taken under suspicion of PC in WL or AFI mode and the histological detection rate. Under WL, 38 biopsies were taken from potentially malignant lesions. In WL mode, 25 of the 38 biopsies were positive for malignancy (66\%). In AFI mode, 29 of the 38 biopsies were suspicious for tumor, meaning that the whitish peritoneal lesions turned purple under AFI; 25 of these 29 suspicious areas were proved to be malignant. This resulted in a neoplasm detection rate of $86 \%$ using AFI. Of the 38 biopsies, 9 showed no color change, indicating fibrotic or necrotic peritoneal tissue. 
Table 3. Morphology and Histological Confirmation Rate of Suspicious Lesions Taken in White Light and Autofluorescence Mode.

\begin{tabular}{|c|c|c|c|c|}
\hline \multirow[b]{3}{*}{ Morphology } & & & \multicolumn{2}{|c|}{ Autofluorescence Mode } \\
\hline & \multicolumn{2}{|c|}{ White Light Mode } & \multirow{2}{*}{$\frac{\text { Tumor Suspicious }}{\mathrm{n}, \text { Histo pos }}$} & \multirow{2}{*}{$\frac{\text { Not Tumor Suspicious }}{\text { n, Histo pos }}$} \\
\hline & $\mathrm{n}$ & $\mathrm{n}$, Histo pos & & \\
\hline Whitish color & 38 & $25(66 \%)$ & $29,25(86 \%)$ & $9,4(44 \%)$ \\
\hline Node & 20 & $13(65 \%)$ & $17,16(94 \%)$ & 3,1 \\
\hline Plane & 18 & $12(67 \%)$ & $12,9(75 \%)$ & 6,3 \\
\hline
\end{tabular}

Abbreviations: Histo, histology; pos, positive.

Table 4. Number of High- and Low-AF-Intensity Lesions. ${ }^{a}$

\begin{tabular}{|c|c|c|c|c|c|c|}
\hline & \multicolumn{2}{|c|}{ High AF } & \multicolumn{2}{|c|}{ Low AF } & \multicolumn{2}{|c|}{ Total (High and Low AF) } \\
\hline & $\mathrm{n}$ & Tumor pos & $\mathrm{n}$ & Tumor pos & $\mathrm{n}$ & Tumor pos \\
\hline Nodule lesion & 6 & 6 & II & 10 & 17 & $16(94 \%)$ \\
\hline Flat lesion & 6 & 4 & 6 & 5 & 12 & $9(75 \%)$ \\
\hline Total & 12 & $10(83 \%)$ & 17 & I5 (88\%) & & \\
\hline
\end{tabular}

Abbreviations: AF, autofluorescence; pos, positive.

${ }^{a}$ Correct identification of malignancy rate is more in nodules ( $\left.94 \%\right)$ compared with flat peritoneal lesions (75\%).

The histological evaluation of these 9 biopsies showed cancer in 4 of the 9 biopsies (44\%; Table 3).

\section{Classification of Lesions Using AF}

The tumor detection rate was slightly higher in low-AF as compared with high-AF lesions ( 83 vs $88 \%$ ). Moreover, the tumor-positive rate was higher in nodules (94\%) compared with flat peritoneal lesions $(75 \%)$. The results are shown in Table 4.

\section{Sensitivity and Specificity}

For AFI, sensitivity was $25 / 29(86 \%)$ and specificity $5 / 8$ (62.5\%). The positive predictive value was 25/28 (0.89), and the negative predictive value $5 / 9(0.55)$. In nodular lesions alone, we found a sensitivity of $16 / 17$ (94\%) and a specificity of $2 / 2(100 \%)$. In flat lesions, the sensitivity was $9 / 12(75 \%)$ and specificity $3 / 6(50 \%)$.

Because all biopsies taken during WL laparoscopy were considered to be cancer positive, calculation of sensitivity, specificity, and negative predictive value is impossible. The overall results of WL are 25/38 (0.65) for positive predictive value (Table 2).

\section{Intraoperative Findings and Postoperative Consequence}

Conventional WL and AFI evaluation of conspicuous peritoneal lesions was successful in all 10 patients. As shown in Figure 1, in the different gastrointestinal tumors and in the ovarian cancer, the $\mathrm{AF}$ of tumor-suspicious lesions resulted in a purple color change. AF images can be further processed. The ratio of green fluorescence and blue background fluorescence gives G/B values, which allow us to create pseudocolor images using ImageJ (http://rsbweb.nih.gov/ij/). This may ease the visual detection of tumors. However, this was not done during this study, and only a sample image is given (Supplemental Figure S1).

Out of the 10 patients, 6 were not selected for the CRS + HIPEC procedure because of a diffuse tumor burden of the small intestine. Of the remaining patients, 3 underwent CCR-0 resection. In 1 patient, open surgery revealed lymph node metastases in the mesentery of the small intestine and more intra-abdominal tumor load than expected, so that only palliative surgery could be performed.

\section{Discussion and Conclusion}

AFI has been implemented for detection of bronchial dysplasias and malignant tumors. These studies suggest that the use of fluorescence bronchoscopy in addition to conventional WL examination could significantly enhance the detection of bronchial lesions. ${ }^{22,23}$ AFI is a novel technology and may be a valuable tool for detection and diagnosis of colorectal neoplastic lesions during endoscopic examination of the gastrointestinal tract. ${ }^{13-15}$

Until now, AFI technology has never been used in patients with PC. Therefore, the objective of this study 


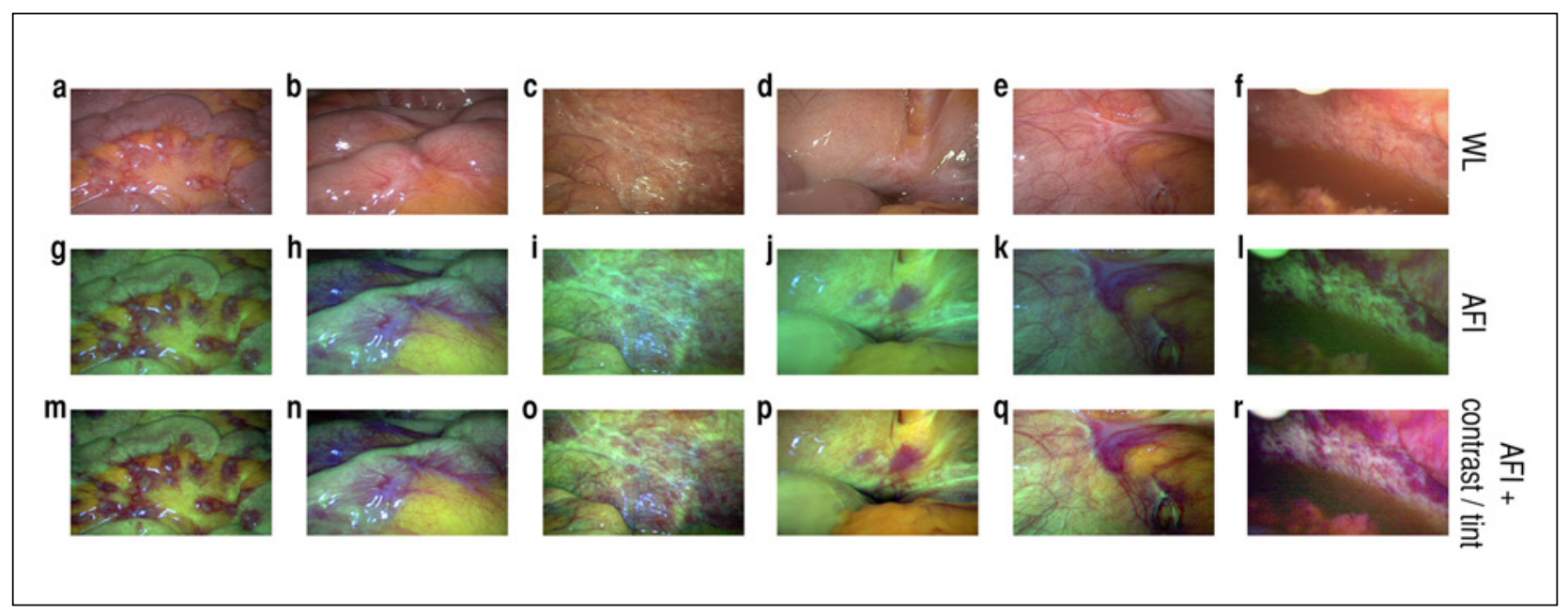

Figure I. Intraoperative findings using $\mathrm{WL}$ and $\mathrm{AFI}{ }^{\mathrm{a}}$

Abbreviations: WL, white-light laparoscopy; AFI, autofluorescence imaging; PC, peritoneal carcinomatosis.

allustration of intraoperative pictures of PC under WL (first line) and corresponding pictures in AF mode (second line). Laparoscopic magnification was 2.5 -fold. AF pictures are corrected for contrast and tint in line 3. Columns of pictures (a), (b), and (c) are examples for PC from colon cancer with clear demarcation of tumor-involved areas in AF mode. Pictures (c) and (i) show partly nodular and partly flat peritoneal lesions. AFI shows partial tumor involvement. The column of picture (d) shows a diffuse whitish plane and thin peritoneal changes in a patient with gastric cancer. AF shows a clear demarcation of the potential tumor area. AF identification of a malignant process in an apparent adhesion in picture (e). The last column shows the abdominal wall with slight whitish peritoneal changes and ascites under WL (e) in a peritoneal mucinous carcinomatosis of the intermediate group. Improved visualization of pathological changes by AFI (I).

was to evaluate the feasibility of adding AFI to WL in peritoneal metastases. Because the concept of the study was designed to evaluate the feasibility of AFI in PC, we compared the accuracy of detecting tumor-suspicious peritoneal lesions under $\mathrm{WL}$ and $\mathrm{AF}$ conditions. It was not the aim of this pilot study to identify any lesions missed under WL examination.

The correct prediction of the histological result was superior using AFI compared with WL only ( 86 vs $66 \%$ ). These results agree with the higher detection rate of pathological lesions under AFI described in bronchoscopy, colonoscopy, and laparoscopy in patients with endometriosis. $^{7,13,15,23,24}$ In all the 3 tumor entities as well as in the intermediate type I of the mucinous appendiceal tumor, tumor-positive spots could clearly be detected and biopsied in AF mode. AFI appears to offer potential for improving the detection rate of malignant foci in $\mathrm{PC}$ of colon, gastric, and ovarian cancer.

In the present investigation, the frequency of correct prediction of malignant histology was approximately balanced under WL in nodular and flat peritoneal lesions (70\% and $75 \%$ ). Using AFI, $94 \%$ of the nodular lesions and only $75 \%$ of the flat lesions were correctly categorized as malignant. Therefore, we suggest biopsy of nodular areas rather than flat regions. Similar results were found in other studies of colonoscopy in patients with chronic inflammatory bowel disease. ${ }^{14,25,26}$

Regarding AF intensity, we found a trend toward higher prevalence of dysplasia in lesions with low $\mathrm{AF}$ than in those with high AF. This may partially be explained by the fact that in nodular areas, the tumor itself is thicker than in flat lesions. Thus, AF emission is rather low. Because 8 of the tumors were classified as G3, 1 tumor as G2, and 1 tumor as intermediate type I, we are unable to state if low $\mathrm{AF}$ is correlated to higher tumor grading.

For all biopsies, sensitivity of AFI was $86 \%$ and specificity $62.5 \%$. Both parameters clearly increased when only nodular lesions were evaluated (sensitivity $=94 \%$; specificity $=100 \%)$. In contrast, sensitivity $(75 \%)$ and specificity $(50 \%)$ were lower in flat lesions. We conclude that AFI is useful in the detection of small tumor nodules. The reduced sensitivity and specificity in flat lesions warrants further study. Matsumoto et $\mathrm{al}^{14}$ found similar results for flat lesions in AFI colonoscopy with a good sensitivity but poor specificity. They speculated that AFI may identify active inflammation as well as neoplastic areas. Similar phenomena may have been present in our study. Therefore, it appears essential to verify the intraoperative tumor diagnosis by frozen section. The clinical evaluation of additional lesions may be improved after 1 histologically positive nodule can be used as a comparator. Selecting the correct nodule for biopsy is crucial in PC patients with suspicion of cancer of the small intestine. Widespread involvement of the small intestine is the most frequent reason for considering a cancer as nonresectable. ${ }^{27}$ In the case of histological verification of malignancy in 1 suspicious lesion using the AF mode, it 
is very probable that similar lesions under $\mathrm{AF}$ mode are also tumor positive. Therefore, small-bowel lesions can be correctly categorized without the risk of perforation after biopsy. Furthermore, the high sensitivity and specificity of the prediction of malignancy in tumor nodules evaluated using AFI may help in selecting informative areas for biopsy. This may reduce the number of biopsies needed and, thus, histopathological costs.

In our study, the time interval between chemotherapy and conventional WL and AFI evaluation was at least 2 weeks. No sufficient data for an evidence-based statement regarding the potential influence of chemotherapy on AFI exist. Probably chemotherapy would induce necrosis of tumor tissue resulting in white foci, which in turn would not be biopsied under AFI guidance.

Our data suggest that laparoscopy in patients with PC is a safe procedure, confirming evidence from other investigators. ${ }^{27-29}$ Adding AFI to the operative procedure most likely does not affect morbidity and mortality.

In summary, one potential application for WL and AFI may be early recognition of $\mathrm{PC}$ in asymptomatic patients at high risk for developing PC. Some authors reported more than 50\% recurrences in the first year after CRS and perioperative intraperitoneal chemotherapy in such groups of patients. ${ }^{30,31}$ In these studies, PC was mostly diagnosed by laparotomy. In our opinion, WL in combination with AFI might be a better method to detect early inconspicuous $\mathrm{PC}$ in high-risk patients.

Laparoscopic AFI evaluation is feasible in patients with PC. The application of AFI in laparoscopy and proper interpretation of the visual impressions requires training but facilitates real-time evaluation in most peritoneal lesions. The addition of AFI to WL may be a promising novel technology in the detection of neoplastic peritoneal foci. The results of this pilot study provide a rationale for further investigation of this technique in larger clinical trials.

\section{Declaration of Conflicting Interests}

The author(s) declared no potential conflicts of interest with respect to the research, authorship, and/or publication of this article.

\section{Funding}

The author(s) received no financial support for the research, authorship, and/or publication of this article.

\section{References}

1. Levy AD, Shaw JC, Sobin LH. Secondary tumors and tumorlike lesions of the peritoneal cavity: imaging features with pathologic correlation. Radiographics. 2009;29: 347-373.

2. Glehen O, Kwiatkowski F, Sugarbaker PH, et al. Cytoreductive surgery combined with perioperative intraperitoneal chemotherapy for the management of peritoneal carcinomatosis from colorectal cancer: a multi-institutional study. J Clin Oncol. 2004;22:3284-3292.

3. Elias D, Lefevre JH, Chevalier J, et al. Complete cytoreductive surgery plus intraperitoneal chemohyperthermia with oxaliplatin for peritoneal carcinomatosis of colorectal origin. J Clin Oncol. 2009;27:681-685.

4. Sugarbaker PH. Intraperitoneal chemotherapy and cytoreductive surgery for the prevention and treatment of peritoneal carcinomatosis and sarcomatosis. Semin Surg Oncol. 1998;14:254-261.

5. Haringsma J, Tytgat GN, Yano H, et al. Autofluorescence endoscopy: feasibility of detection of GI neoplasms unapparent to white light endoscopy with an evolving technology. Gastrointest Endosc. 2001;53:642-650.

6. Vo-Dinh T, Panjehpour M, Overholt BF, Farris C, Buckley FP III, Sneed R. In vivo cancer diagnosis of the esophagus using differential normalized fluorescence (DNF) indices. Lasers Surg Med. 1995;16:41-47.

7. Buchweitz O, Staebler A, Tio J, Kiesel L. Detection of peritoneal endometriotic lesions by autofluorescence laparoscopy. Am J Obstet Gynecol. 2006;195:949-954.

8. Kara MA, Bergman JJ. Autofluorescence imaging and narrow-band imaging for the detection of early neoplasia in patients with Barrett's esophagus. Endoscopy. 2006;38:627-631.

9. Kara MA, Peters FP, Fockens P, ten Kate FJ, Bergman JJ. Endoscopic video- autofluorescence imaging followed by narrow band imaging for detecting early neoplasia in Barrett's esophagus. Gastrointest Endosc. 2006;64:176-185.

10. Li BH, Xie SS. Autofluorescence excitation-emission matrices for diagnosis of colonic cancer. World J Gastroenterol. 2005;11:3931-3934.

11. Yeung TM, Mortensen NJ. Advances in endoscopic visualization of colorectal polyps. Colorectal Dis. 2011;13:352-359.

12. Ahmed T, Monti J, Lashner B. Random versus targeted biopsies for colorectal cancer surveillance in inflammatory bowel disease. Gastroenterol Hepatol (N Y). 2010;6: 438-442.

13. Inoue K, Wakabayashi N, Morimoto Y, et al. Evaluation of autofluorescence colonoscopy for diagnosis of superficial colorectal neoplastic lesions. Int J Colorectal Dis. 2010;25:811-816.

14. Matsumoto T, Nakamura S, Moriyama T, Hirahashi M, Iida M. Autofluorescence imaging colonoscopy for the detection of dysplastic lesions in ulcerative colitis: a pilot study. Colorectal Dis. 2010;12:e291-e297.

15. Takeuchi Y, Inoue T, Hanaoka N, et al. Autofluorescence imaging with a transparent hood for detection of colorectal neoplasms: a prospective, randomized trial. Gastrointest Endosc. 2010;72:1006-1013.

16. Haussinger K, Becker H, Stanzel F, et al. Autofluorescence bronchoscopy with white light bronchoscopy compared with white light bronchoscopy alone for the detection of precancerous lesions: a European randomised controlled multicentre trial. Thorax. 2005;60:496-503.

17. Zheng W, Lau W, Cheng C, Soo KC, Olivo M. Optimal excitation-emission wavelengths for autofluorescence diagnosis of bladder tumors. Int J Cancer. 2003;104:477-481. 
18. Ronnett BM, Zahn CM, Kurman RJ, Kass ME, Sugarbaker $\mathrm{PH}$, Shmookler BM. Disseminated peritoneal adenomucinosis and peritoneal mucinous carcinomatosis: a clinicopathologic analysis of 109 cases with emphasis on distinguishing pathologic features, site of origin, prognosis, and relationship to "pseudomyxoma peritonei." Am J Surg Pathol. 1995;19:1390-1408.

19. Matsuda T, Saito Y, Fu KI, et al. Does autofluorescence imaging videoendoscopy system improve the colonoscopic polyp detection rate? A pilot study. Am J Gastroenterol. 2008;103:1926-1932.

20. Matsumoto T, Moriyama T, Yao T, Mibu R, Iida M. Autofluorescence imaging colonoscopy for the diagnosis of dysplasia in ulcerative colitis. Inflamm Bowel Dis. 2007; 13:640-641.

21. van den Broek FJ, Fockens P, van Eeden S, et al. Endoscopic tri-modal imaging for surveillance in ulcerative colitis: randomised comparison of high-resolution endoscopy and autofluorescence imaging for neoplasia detection; and evaluation of narrow-band imaging for classification of lesions. Gut. 2008;57:1083-1089.

22. Haussinger K, Stanzel F, Huber RM, Pichler J, Stepp H. Autofluorescence detection of bronchial tumors with the D-light/AF. Diagn Ther Endosc. 1999;5:105-112.

23. Shibuya K, Fujisawa T, Hoshino H, et al. Fluorescence bronchoscopy in the detection of preinvasive bronchial lesions in patients with sputum cytology suspicious or positive for malignancy. Lung Cancer. 2001;32:19-25.

24. Moro-Sibilot D, Jeanmart M, Lantuejoul S, et al. Cigarette smoking, preinvasive bronchial lesions, and autofluorescence bronchoscopy. Chest. 2002;122: 1902-1908.

25. Blonski W, Kundu R, Lewis J, Aberra F, Osterman M, Lichtenstein GR. Is dysplasia visible during surveillance colonoscopy in patients with ulcerative colitis? Scand $J$ Gastroenterol. 2008;43:698-703.

26. Rubin DT, Rothe JA, Hetzel JT, Cohen RD, Hanauer SB. Are dysplasia and colorectal cancer endoscopically visible in patients with ulcerative colitis? Gastrointest Endosc. 2007;65:998-1004.

27. Pomel C, Appleyard TL, Gouy S, Rouzier R, Elias D. The role of laparoscopy to evaluate candidates for complete cytoreduction of peritoneal carcinomatosis and hyperthermic intraperitoneal chemotherapy. Eur J Surg Oncol. 2005;31:540-543.

28. Contreras CM, Stanelle EJ, Mansour J, et al. Staging laparoscopy enhances the detection of occult metastases in patients with pancreatic adenocarcinoma. J Surg Oncol. 2009;100:663-669.

29. Garofalo A, Valle M. Laparoscopy in the management of peritoneal carcinomatosis. Cancer J. 2009;15:190-195.

30. Elias D, Honore C, Dumont F, et al. Results of systematic second-look surgery plus HIPEC in asymptomatic patients presenting a high risk of developing colorectal peritoneal carcinomatosis. Ann Surg. 2011;254:289-293.

31. Bijelic L, Yan TD, Sugarbaker PH. Failure analysis of recurrent disease following complete cytoreduction and perioperative intraperitoneal chemotherapy in patients with peritoneal carcinomatosis from colorectal cancer. Ann Surg Oncol. 2007;14:2281-2288. 